\title{
Suizid bei Krankheit
}

\author{
Josef Widlera, Michael Kohlbacher ${ }^{b}$ \\ ${ }^{\text {a }}$ Dr. med., Präsident AGZ; ${ }^{b}$ Dr. iur., MPH, Generalsekretär AGZ
}

Die SAMW hat nach einer öffentlichen Vernehmlassung am 6. Juni 2018 «Medizinethische Richtlinien - Umgang mit Sterben und Tod» veröffentlicht. Neu sollen Ärzte Beihilfe zum Suizid bei nicht tödlichen Krankheiten leisten können. Damit werden die Grenzen ärztlichen Handelns überschritten. Aus Sicht der Selbstbestimmung der Patienten wäre das nicht nötig. Es bräuchte ein neues Verfahren, und der Staat muss seine Rolle wahrnehmen.

\section{Problematik des Vorgehens}

Die SAMW legt sich in ihren neuen Richtlinien in einer für den ärztlichen Berufsstand zentralen standesrechtlichen Frage gegen die Meinung des Zentralvorstands der FMH fest. Sie beruft sich auf die überwiegende Mehrheit positiver Stellungnahmen im Rahmen einer öffentlichen Vernehmlassung sowie darauf, dass nicht der Zentralvorstand, sondern die Ärztekammer über die Übernahme der Richtlinien in das Standesrecht der Ärzte zu entscheiden habe. Das ist aus formaler Sicht richtig. Das Vorgehen der SAMW, das ärztliche Standesrecht in dieser wesentlichen Frage über den Weg einer öffentlichen Vernehmlassung vorherzubestimmen, anstatt mit der Standesorganisation eine gemeinsame Lösung zu erarbeiten, wirft aber Zweifel auf. Angesichts der Tragweite der Thematik für das ärztliche Handeln sollte ein allfälliger Beschluss der Ärztekammer, wie auch immer er ausgeht, einer Urabstimmung unterzogen werden.

\section{Begründung der Ausweitung ärztlicher Suizidhilfe gemäss SAMW}

Die neuen «Medizinisch-ethischen Richtlinien - Umgang mit Sterben und Tod» erklären unter bestimmten Voraussetzungen die ärztliche Suizidhilfe bei Krankheiten oder Funktionseinschränkungen, die nicht zum Tod führen, als vertretbar. Die Nicht-Eingrenzung der Suizidhilfe auf tödliche Krankheiten wird damit begründet, dass diese nicht objektivierbar seien respektive Patienten und Behandelnde unter Rechtfertigungsdruck geraten könnten, wenn sie bei Vorliegen einer tödlichen Krankheit die Möglichkeit der Suizidhilfe nicht in Betracht ziehen wollen. Meist sei es auch nicht ein medizinisch objektivierbarer Zustand, der bei Patienten zum Suizidwunsch führe, sondern das subjektiv erlebte unerträgliche Leiden [1].

Es ist schon erstaunlich, dass und wie die Öffnung der ärztlichen Suizidhilfe mit «schwieriger Objektivierbarkeit» tödlicher Krankheiten und «Rechtfertigungsdruck» im Falle einer Verweigerung von Suizidhilfe begründet wird. Es soll stattdessen genügen, ein ganz und gar nicht objektivierbares, von Patienten subjektiv empfundenes Leiden "im intersubjektiven Nachvollzug durch die behandelnde Ärztin plausibel zu machen». Was die SAMW darunter versteht, welche Methodik beim Nachvollzug eines Leidens anzuwenden und welche Kompetenzen dazu notwendig wären, verschweigt die SAMW. So entsteht der Eindruck eines "Jekami», und das darf wohl angesichts der Tragweite der Entscheide für ärztliche Suizidhilfe nicht der Fall sein.

Ein «Rechtfertigungsdruck» bei der Verweigerung der Suizidhilfe im Kontext von Leiden dürfte sogar noch grösser werden respektive die "Erlösung vom Leiden» vielmehr als «humanitärer» Akt dargestellt werden, wie es auch die Vizepräsidentin von EXIT jüngst getan hat [2].

\section{Leiden im Kontext des Krankheits- verständnisses}

Es stellt sich die Frage, von welchem Verständnis von Krankheit und Gesundheit man ausgeht, wenn die Bewertung eines subjektiv empfundenen Leidens Entscheidungsgrundlage für ärztliche Suizidhilfe sein soll. Ärzte beurteilen Krankheiten überwiegend anhand von objektiven, biomedizinisch messbaren Kriterien. Gerade bei der Beurteilung des subjektiven Erlebens eines objektiv messbaren Krankheitsbildes sind jedoch andere Konzepte und Modelle zu berücksichti- 
gen, die die soziale Dimension von Krankheit und Gesundheit bzw. von Widerstandsressourcen mit einbeziehen. Anhand dieser Modelle hat sich ein Verständnis dafür herausgebildet, dass Menschen angesichts schwerer Krankheiten länger leben bzw. ein Leiden eher als erträglich empfinden, wenn sie über gewisse materielle, soziale, psychische und seelische Ressourcen verfügen.

Auch der Sterbewunsch sozial isolierter Personen oder von Personen, die angesichts von Krankheiten über geringe psychosoziale Ressourcen verfügen, ist zu respektieren. Eine, wenn auch nur unbewusste, "soziale Selektion" darf aber nie passieren. Suizidhilfe darf daher nie ohne seriöse Abklärung von nicht medizinisch beeinflussbaren, psychosozialen Faktoren bzw. von Unterstützungsmöglichkeiten zur Verbesserung der Situation geleistet werden. Darauf gehen die Richtlinien nicht ein.

\section{Selbstbestimmungsrecht auf Suizidhilfe und Rolle der Ärzte}

Das Selbstbestimmungsrecht im Zusammenhang mit der Beendigung des Lebens durch Suizid ist durch die Verfassung und die Europäische Menschenrechtskonvention geschützt und vom Bundesgericht in seinem Urteil vom 3. November 2006 bestätigt. Das verfassungsrechtlich geschützte Recht auf Leben verpflichtet aber den Staat im Zusammenhang mit Suizidhilfe durch ein gefährliches Betäubungsmittel dazu, durch ein verlässliches Kontrollverfahren sicherzustellen, dass der Entscheid tatsächlich dem freien und wohlerwogenen Willen des Betroffenen entspricht [3].

Mit der Rezeptpflicht für Natrium-Pentobarbital (NaP) überträgt der Staat seine Kontrollpflicht auf die Ärzte. Die ärztliche Rezeptur ist in diesem Kontext keine medizinische Handlung, sondern eine vom Staat delegierte Kontrollmassnahme zur Vermeidung des missbräuchlichen Einsatzes von NaP. Die Rezeptur der tödlichen Dosis von NaP bei nicht tödlichen Krankheiten ist hingegen rechtlich fragwürdig und verstösst gegen die Grundsätze ärztlichen Handelns: denn die Aufgabe der Ärzte ist es, menschliches Leben zu schützen, Gesundheit zu fördern und zu erhalten, Krankheiten zu behandeln, Leiden zu lindern und Sterbenden beizustehen [4]. Ärztliche Aufgabe kann es sein, bei einem bereits eingetretenen oder unausweichlich bevorste- henden Sterbeprozess das Sterben zu erleichtern oder zu verkürzen, auch durch Zustimmung zur Abgabe von NaP. Aufgabe von Ärzten darf es nicht sein, mit der Verordnung der tödlichen Dosis von NaP aktiv dazu beizutragen, dass ein Leben beendet wird, das ansonsten infolge einer Krankheit oder Funktionseinschränkung nicht zum Ende führen würde.

\section{Ärztliche oder staatlich geregelte Suizidhilfe - wie weiter?}

Ärzte sollen weiterhin eine Rolle übernehmen, wenn es um die Beurteilung eines freien, selbstbestimmten Entscheides im Zusammenhang mit Sterbewünschen von Patienten geht. Vor allem für die Beurteilung der Urteilsfähigkeit und der Selbstbestimmung bei Sterbewünschen im Kontext von nicht tödlichen Krankheiten muss aber ein anderer Weg gefunden werden, als von der SAMW nunmehr vorgeschlagen wird. Der Staat muss das Selbstbestimmungsrecht der Menschen gewährleisten und seine Verantwortung übernehmen, sie durch ein geeignetes Verfahren vor übereilten Entscheidungen und Missbrauch zu schützen. Das gilt besonders bei Suizidwünschen angesichts von nicht tödlichen Krankheiten

Die vom Bundesgericht geforderte Unabhängigkeit der Patienten gegenüber fremden Einflüssen sollte auch gegenüber behandelnden Ärzten gelten. Es wäre ein neues Verfahren zur Beurteilung selbstbestimmter Sterbewünsche von Patienten zu definieren, z.B. durch die besondere Form einer von neutralen Personen bezeugten letztwilligen Verfügung des Patienten und die Begutachtung der Urteilsfähigkeit durch eine unabhängige Person oder Stelle.

Für die Abgabe der tödlichen Dosis von NaP wäre sodann eine kontrollierte Abgabestelle zu schaffen, bei der die Patienten die Dosis aufgrund der Bescheinigung der Gutachterstelle beziehen können - ein ärztliches Rezept bräuchte es dafür nicht.

\section{Literatur}

1 SAMW-Richtlinien, Kapitel 6.2, S. 25; Kind C. Umgang mit Sterben und Tod. Schweiz Ärzteztg. 2018;99(24):791.

2 Marion Schafroth, "Suizidhilfe - ein humanitärer Akt», Tages-Anzeiger, 16. Juni 2016

3 BGE 133 I 58, Erwägungen 6.1-6.3.

4 Standesordnung FMH, Art. 2. 